\title{
Automatic multi-organ segmentation of prostate magnetic resonance images using watershed and nonsubsampled contourlet
}

\section{transform}

\author{
Zhe Huang, ${ }^{a}$ Shan Jiang, ${ }^{\text {a }}$ Zhiyong Yang, ${ }^{a}$ Yabin Ding, ${ }^{a}$ Wei Wang, ${ }^{\text {}}$ Yan $Y u^{c}$ \\ ${ }^{a}$ Tianjin University, Center for Advanced Mechanisms and Robotics, School of Mechanical Engineering, 92 \\ Weijin Road, Nankai District, Tianjin, China, 300072 \\ ${ }^{\mathrm{b}}$ Tianjin Medical University Cancer Institute and Hospital, Huanhuxi Road, Hexi District, Tianjin, China, \\ 300060 \\ cDivision of Medical Physics, Department of Radiation Oncology, Thomas Jefferson University, Kimmel \\ Cancer Center, 111 South 11th Street, Philadelphia, PA 19107
}

\begin{abstract}
The watershed is an efficient algorithm for the segmentation of images. However, over-segmentation, which contains so many tiny regions that regions of interest cannot be identified easily, decreases the effectiveness. In this paper, pre-processing of images and the modification of watershed algorithm are both studied to restrain the over-segmentation. In the process of pre-processing, a kind of multi-scaled transform, contrast à trous wavelet based contourlet transform, is proposed and constructed to get sparse representation. In the aspect of modifying watershed, the "texture gradient" is defined, and the texture gradient is combined with marker-based watershed algorithm to reduce the number of segmented regions. The proposed method is tested by 36 prostate MR images and compared with several image segmentation algorithms; the experiment and comparison results show that the proposed method consistently restrains the number of segmented regions. The segmentation results correctly correspond to the main tissues in the images, and each tissue is integrally segmented respectively with the elimination of small regions. The segmentation accuracy rate is $87.29 \%$, which is higher than other methods under comparison.
\end{abstract}

Keywords: prostate magnetic resonance image; multi-organ segmentation; multi-scaled geometric image analysis; watershed.

Address all correspondence to: Shan Jiang, Tianjin University, Center for Advanced Mechanisms and Robotics, School of Mechanical Engineering, 92 Weijin Road, Nankai District, Tianjin, China, 300072;Tel: +86 13212154030; Fax: +86-022-27401042; E-mail: shanjmri@tju.edu.cn

\section{Introduction}

Prostate cancer is one of the most universal malignant tumors in the male reproductive system and a main kind of disease which causes of death for men ${ }^{1}$. Transperineal iodine-125 (125I) seed implantation is an effective approach for the treatment of localized prostate cancers, and the particle treatment planning plays an important role during the treatment ${ }^{2}$. In order to obtain precise treatment planning, virtual three-dimensional 
models of prostate and tissues around the target are generated in computer, and based on the model, particle treatment planning is set. Dose volume histogram (DVH) is used to evaluate and optimize the plan, which can cure prostate cancer and avoid damaging tissues around the prostate. The precision of models constructed based on image segmentation is the precondition of the seed implantation treatment. Because of the high contrast, MR images are chosen to perform the pretreatment of prostate cancer ${ }^{3}$. Therefore, a robust automatic segmentation of multi-organ technique for prostate MR images plays an important role in image guided brachytherapy treatment of prostate cancer.

However, automatic tissue classification of MR images is still a challenging task in applications ${ }^{4}$. The watershed segmentation ${ }^{5}$ is an efficient tool for image classification and has been widely used in medical image segmentation and improved by combined with some classical algorithm ${ }^{6}$. The method regards image as a 3D topography surface, starts the region growing from the surface minima detected by image gradient, combines both the discontinuity and similarity properties effectively and finally obtains the segmentation results of one pixel wide, closed connected precise contours of objects ${ }^{7}$. Using gradient information of the actual image, watershed segmentation obtains accurate results for images containing connecting objects. The main courses of the algorithm are smoothing and extracting gradient image from the original image. However, the segmentation method has a major problem of over-segmentation $^{8}$, as shown in Fig. 1. (b), where so many tiny regions are generated that regions of interest cannot be identified easily.

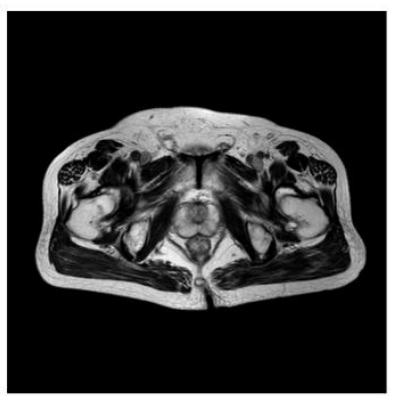

(a)

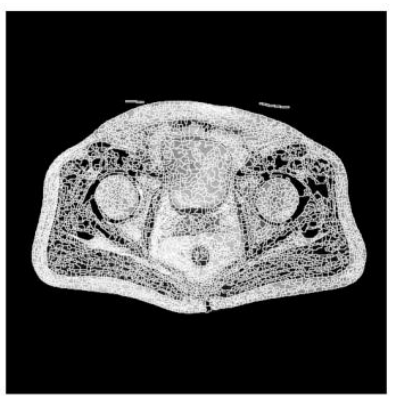

(b)

Fig. 1 Over-segmentation caused by watershed. (a) Original image, (b) The segmentation results of watershed only. The original image is over-segmented.

There are two main methods to restrain the over-segmentation of watershed. One way is to perform pre-processing before segmentation, such as obtaining sparse representation of original images ${ }^{9}$, the other is to improve the watershed segmentation, such as marker-controlled watershed-based segmentation ${ }^{10}$. In this paper, two methods are studied. In the process of pre-processing, a new kind of multi-scaled transform, contrast à trous wavelet based contourlet transform, is proposed and constructed to get sparse representation. In the aspect of modifying watershed, the concept of the "texture gradient" and marker-based watershed segmentation are introduced, and the texture gradient is used in marker-based watershed to reduce the number of segmented regions. The main procedures of the proposed method are shown in Fig.2. First, original images are performed the decomposition of contrast à trous wavelet based contourlet transform, and low-frequency images and high-frequency images are separated. Through the transform, some spurious minimal values 
widely exists in medical images are removed. Then, the low-frequency images are segmented by the marker-based watershed segmentation using texture gradient. Although images in level 2 in Fig. 2 are not clear in visual aspect, they make contribution to the final segmentation result. The results achieved in this step are low resolution image, which is vaguer than original image. Finally, the inverse contourlet transform is implemented to get high resolution image. The achieved image is the final segmentation results. In order to facilitate reconstructing three-dimensional model and enhance the intuition of the model, the segmentation results are labeled with special numbers and colored by different colors. Based on a series of segmentation results, the three-dimensional model can be reconstructed.

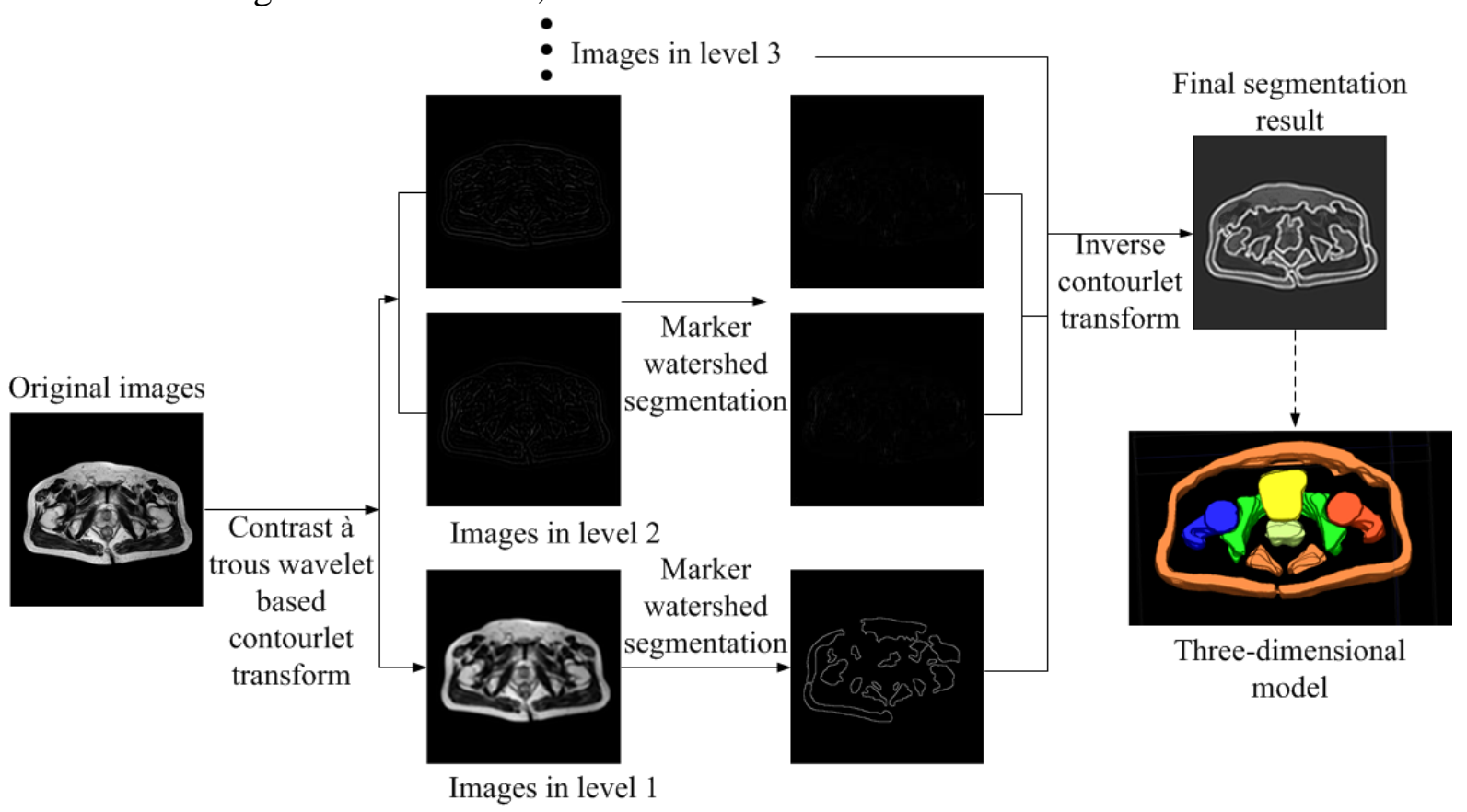

Fig. 2 Flowchart of the proposed segmentation method.

Through these processes mentioned above, the over-segmentation of watershed is efficiently reduced. The segmentation has been evaluated using prostate MR images. The achieved results are more continuous and satisfying as a result of the human organs. According to the experiment results, this method lends itself well to the multi-organ segmentation.

This paper is arranged as follows: Section 2 introduces in detail the contourlet transform and the way implementing image analysis by contrast à trous wavelet based contourlet transform and directional filter bank. In section 3, "stationary texture gradient" and its combination with watershed algorithm are addressed, the integration of marker watershed segmentation and contrast à trous wavelet based contourlet transform is also proposed. In section 4, experimental results and related analysis are presented. Finally, the whole paper is concluded.

\section{Contrast à Trous Wavelet Based Contourlet Transform}

The watershed segmentation has been widely used in medical image segmentation. Although the method is efficient, the segmentation is often over-segmented. In order to restrain the over-segmentation, contourlet transform ${ }^{11}$, which is a new method of two-dimensional image 
representation, is performed before segmentation. The transform effectively reduces the number of segmented regions.

\subsection{Contourlet Transform}

Contourlet transform, also called pyramidal directional filter bank (PDFB), is a multi-resolution, local and multi-directional representation method. Different from wavelet transform ${ }^{12}$, contourlet transform theory is constructed in discrete domain, and expended to continuous domain by M.N. Do and M. Vetterli with multi-resolution framework ${ }^{13}$. The objective of this transform is to obtain sparse representation containing line and surface singularity of the original image. By means of basis analogous to contour, the support of the basis has strip structure, whose aspect ratio changing with different scale. Because of the direction and anisotropy of the strip structure, contourlet transform can effectively characterize lines and aspects in the images with sparse representation. The contourlet transform is briefly described in Fig. 3. The contourlet transform consists of the Laplacian pyramid and a directional filter bank.

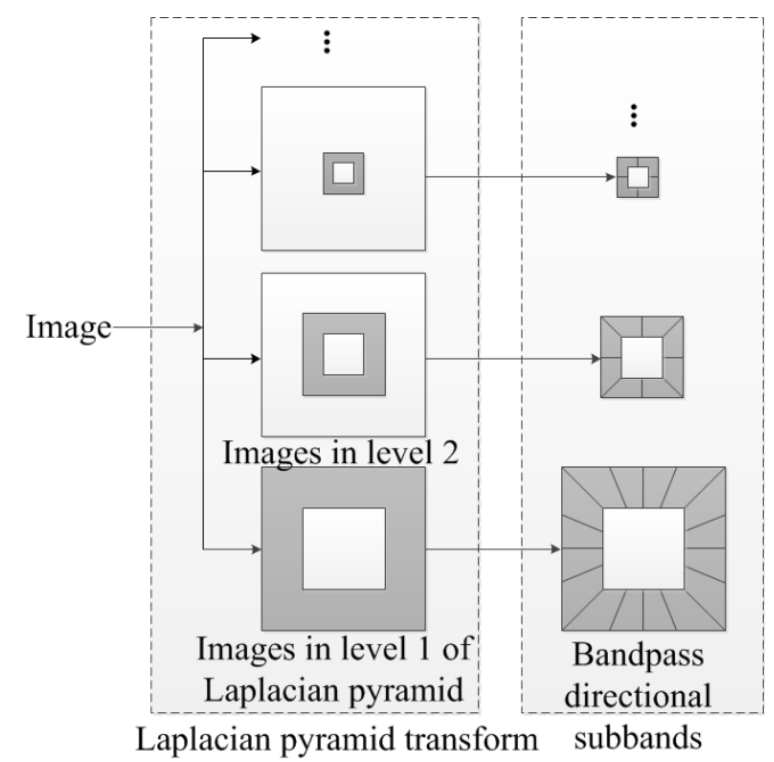

Fig. 3 Flowchart of contourlet transform.

Contourlet transform respectively analyzes multi-scale and direction of an image. First, Laplacian pyramid (LP) is used to implement multi-scale decomposition of each image to detect and capture singular points of the image. In the process of the first decomposition, original image signal is decomposed into low frequencycomponent and high frequency components. And then, the low frequency components are iteratively decomposed to obtain multi-directional image. The high frequency components of each scale are decomposed by directional filter bank (DFB) and the singularities in same direction are comprehensively represented as a coefficient. 


\subsection{Contrast à Trous Wavelet Based Contourlet Transform}

Laplacian pyramid transform is the first step of contourlet transform. However, after performing many experiments and analysis, Alexander Toet ${ }^{14}$ drew the conclusion that multi-resolution contrast pyramid ${ }^{15}$ is more coincident with human vision system (HVS) than Laplacian pyramid transform ${ }^{16}$, and because the terminal purpose of pretreatment is to aid the doctor, the multi-resolution contrast is preferred. Thus, in this paper, the contrast pyramid is constructed to improve the contourlet transform and one of the main algorithms, the ratio of low-pass pyramid, is introduced.

\subsubsection{Contrast Pyramid Transform}

The constitution of contrast pyramid is designed based on standard Laplacian pyramids ${ }^{17}$, which is widely used in images segmentation ${ }^{18}$. The original image is defined as $G_{0}$, and the pixels of $l$ th $(1 \leq l \leq N, N$ is the highest layer $)$ are obtained by:

$$
G_{l}=\operatorname{REDUCE}\left(G_{l-1}\right)
$$

where

$$
G_{i}(i, j)=\sum_{m=-2}^{2} \sum_{n=-2}^{2} \omega(m, n) G_{l-1}(2 i+m, 2 j+n)
$$

The weighting function $\omega(m, n)=\omega^{\prime}(m) \omega^{\prime}(n)$, and $\omega^{\prime}(0)=a, \omega^{\prime}(1)=\omega^{\prime}(-1)=0.5, \omega^{\prime}(2)=\omega^{\prime}(-2)=a / 2, a$ is generally set to 0.375 .

In order to eliminate the difference of pixel density in each layer, interpolation is performed in low frequency images with the EXPAND framework before generating high frequency images. The EXPAND framework can be defined as follows:

$$
\left\{\begin{array}{l}
G_{l, 0}=G_{l} \\
G_{l, k}=\operatorname{EXPAND}\left[G_{l, k-1}\right]
\end{array}\right.
$$

where

$$
G_{l, k}(i, j)=4 \sum_{m=-2}^{2} \sum_{n=-2}^{2} \omega(m, n) G_{l, k-1}\left(\frac{i+m}{2}, \frac{j+n}{2}\right)
$$

and $G_{l, k}(i, j)$ is calculated by the formula when $\frac{i+m}{2}, \frac{j+n}{2}$ is integer, otherwise $G_{l, k}(i, j)=0$.

The ratio of low-pass pyramid can be constructed as follows:

$$
\left\{\begin{array}{l}
R_{l}=\frac{G_{l}}{\operatorname{EXPAND}\left(G_{l+1}\right)}, 0 \leq l \leq N-1 \\
R_{N}=G_{N}
\end{array}\right.
$$

where the $R_{l}$ of each layer is the ratio of images of adjacent two layers.

The reconstitution is the inverse process of constituting ratio low-pass pyramid and the detailed procedure is given: 


$$
\left\{\begin{array}{l}
G_{N}=R_{N} \\
G_{l}=R_{l} \bullet \operatorname{EXPAND}\left(G_{l+1}\right)
\end{array}\right.
$$

where $0 \leq l \leq N-1$. According to the procedure, reconstitution is accomplished.

Then, the contrast of luminance is represented by $C$ and can be defined by:

$$
C=\left(L-L_{b}\right) / L_{b}
$$

where $L$ is the luminance values of some special pixels, $L_{b}$ is the luminance values of local pixels, and for all $i, j, I(i, j)=1$.

Suppose that,

$$
\left\{\begin{array}{l}
C_{l}=\frac{G_{l}}{\operatorname{EXPAND}\left[G_{l+1}\right]}-I, \quad 0 \leq l \leq N-1 \\
C_{N}=G_{N}
\end{array}\right.
$$

Hence, the image sequence represented by $C_{l}$ is contrast pyramid. Because of the integrity of contrast pyramid, original images can be obtained by the inverse transformation of contrast pyramid using formula (9).

$$
\left\{\begin{array}{l}
G_{N}=C_{N} \\
G_{l}=\left(C_{l}+I\right) \bullet \operatorname{EXPAND}\left(G_{l+1}\right)
\end{array}, 0 \leq l \leq N-1\right.
$$

\subsubsection{The Constitution of Contrast à Trous Wavelet Based Contourlet Transform}

The general thought of constitution of contrast à trous wavelet based contourlet transform is to combine the definition of contrast with à trous wavelet transform, and obtain contrast à trous wavelet transform. Then, the transform is used in the contourlet transform.

Because of the undecimated property, à trous wavelet is a well-defined tool for image fusion, pattern recognition and image segmentation. At the same time, contrast pyramid transform is an efficient image representation method. Hence, in this paper, contrast pyramid transform based on contrast à trous wavelet is proposed. First, contrast à trous wavelet is obtained through introducing contrast into traditional à trous wavelet ${ }^{19}$. Second, contrast à trous wavelet is incorporated into contourlet transform to perform multi-scale analysis.

According to à trous wavelet ${ }^{20}$, which is used in image fusion ${ }^{21}$, the filter generated by scaling function (usually $B_{3}$ spine function) is used to implement convolution for original images. For a given image $p$, a series of similar images $p_{l}(l=1,2, \ldots, N, N$ is the number of decomposition layers) are produced by à trous wavelet, and the wavelet plane is obtained by calculating the difference between $p_{l-1}$ and $p_{l}$.

By using contrast pyramid strategy, the contrast of luminance is represented by $C$ and can be defined by:

$$
C=\left(L-L_{b}\right) / L_{b}=\left(L / L_{b}-I\right)
$$

where $L$ is the luminance values of some special pixels, $L_{b}$ is the luminance values of local pixels, and for all $i, j, I(i, j)=1$.

The contrast à trous wavelet transform is defined as follows: 


$$
\left\{\begin{array}{l}
p_{0}=p \\
\omega_{l}=\left(p_{l-1} / p_{l}\right)-I, 0 \leq l \leq N \\
\omega_{N}=p_{N}
\end{array}\right.
$$

where $\omega_{n}$ is the contrast coefficient. Correspondingly, the reconstruction formula can be derived. First, define $\omega_{l}=\left(p_{l-1} / p_{l}\right)-I, l=1,2, \cdots, N, p_{0}=p$, the reconstitution formula are defined as follows:

$$
\left\{\begin{array}{l}
p_{N}=\omega_{N} \\
p_{l-1}=\left(\omega_{l}+I\right) \times p_{l}, 0 \leq l \leq N
\end{array}\right.
$$

Finally, the contourlet transform with contrast à trous wavelet is achieved by replacing the Laplacian Pyramid transform with contrast à trous wavelet.

\subsubsection{DFB Decomposition}

In this paper, decimation-free directional filter bank (DFDFB) is designed to perform the directional decomposition of images. DFDFB is shift invariant by avoiding image sampling and interpolation. In the beginning, a one-dimensional filter is chosen and extended into two-dimensional domain. Then, the two-dimensional filter is transformed to DFDFB. The one-dimensional filter chosen in this paper is half-band filter, which is efficient and robust.

The unit response in sampling cells of half-band filter can be represented as follows:

$$
h(n)= \begin{cases}c, & n=0 \\ 0, & n=2 k(k \neq 0, k=1,2,3 \ldots) \\ h(2 k+1), & n=2 k+1\end{cases}
$$

where $c=1 / 2, h(n)$ is the unit response in sampling cells, $k$ is an integer. The z-transform of is $h(n)$ is performed:

$$
H(z)=c+z^{-1} \sum_{n=0}^{\infty} h(2 n+1) z^{-n}
$$

Suppose that, the frequency response of half-band filter is $H\left(e^{j \omega}\right)$, the amplitude is 1 , and the one-dimensional transform function is $A\left(e^{j \omega}\right)$, then

$$
A\left(e^{j w}\right)=2\left(H\left(e^{j w}\right)-0.5\right)
$$

The one-dimensional filter can be defined as:

$$
B(z)=1+A(z)
$$

The amplitude of $B(z)$ is 2.0 , cut-off frequency is $\pi / 2$. Then, the two-dimensional filter is represented as follows:

$$
\tilde{D}\left(z_{1}, z_{2}\right)=\frac{1}{2} B\left(z_{1}\right) B\left(z_{2}\right)
$$




$$
\begin{gathered}
D\left(z_{1}, z_{2}\right)=\frac{1}{2} B\left(j z_{1}\right) B\left(j z_{2}\right)+\frac{1}{2} B\left(-j z_{1}\right) B\left(-j z_{2}\right) \\
C\left(z_{1}, z_{2}\right)=2-D\left(z_{1}, z_{2}\right)
\end{gathered}
$$

where $D\left(z_{1}, z_{2}\right)$ is the band-pass filter in first and third quadrant, $C\left(z_{1}, z_{2}\right)$ is the band-pass filter in second and forth quadrant.

Fan-shaped filter $H_{\text {fan }}^{1}\left(z_{1}, z_{2}\right)$ in first and third quadrant can be obtained by the transformation: $z_{1} \leftarrow z_{1}^{0.5} z_{2}^{-0.5}, \quad z_{2} \leftarrow z_{1}^{0.5} z_{2}^{0.5} . \quad H_{f a}^{2}\left(z_{1}, z_{2}\right)$ can be calculated as the same transform.

The four-sub-band filter $H_{\text {four }}$ and the first two sub-bands of eight-sub-band $H_{\text {oct }}$ are calculated as follows:

$$
\begin{gathered}
H_{\text {four }}^{1}\left(z_{1}, z_{2}\right)=D\left(z_{1}, z_{2}\right) \bullet H_{\text {fan }}^{2}\left(z_{1}, z_{2}\right) / 2 \\
H_{\text {four }}^{2}\left(z_{1}, z_{2}\right)=D\left(z_{1}, z_{2}\right) \bullet H_{\text {fan }}^{1}\left(z_{1}, z_{2}\right) / 2 \\
H_{\text {four }}^{3}\left(z_{1}, z_{2}\right)=C\left(z_{1}, z_{2}\right) \bullet H_{\text {fan }}^{1}\left(z_{1}, z_{2}\right) / 2 \\
H_{\text {four }}^{4}\left(z_{1}, z_{2}\right)=C\left(z_{1}, z_{2}\right) \bullet H_{\text {fan }}^{2}\left(z_{1}, z_{2}\right) / 2 \\
H_{\text {oct }}^{2}\left(z_{1}, z_{1}\right)=H_{\text {four }}^{1}\left(z_{1}, z_{2}\right) \bullet H_{\text {pshift }}^{1}\left(z_{1}, z_{2}\right) / 2 \\
H_{\text {oct }}^{1}\left(z_{1}, z_{1}\right)=H_{\text {four }}^{1}\left(z_{1}, z_{2}\right)-H_{\text {oct }}^{2}\left(z_{1}, z_{2}\right)
\end{gathered}
$$

Other sub-bans of eight-sub-band $H_{\text {oct }}$ and the sub-band filters with more numbers can be calculated by the same mode. According to the formula presented above, DFB decomposition of the images generated by contrast à trous wavelet is accomplished.

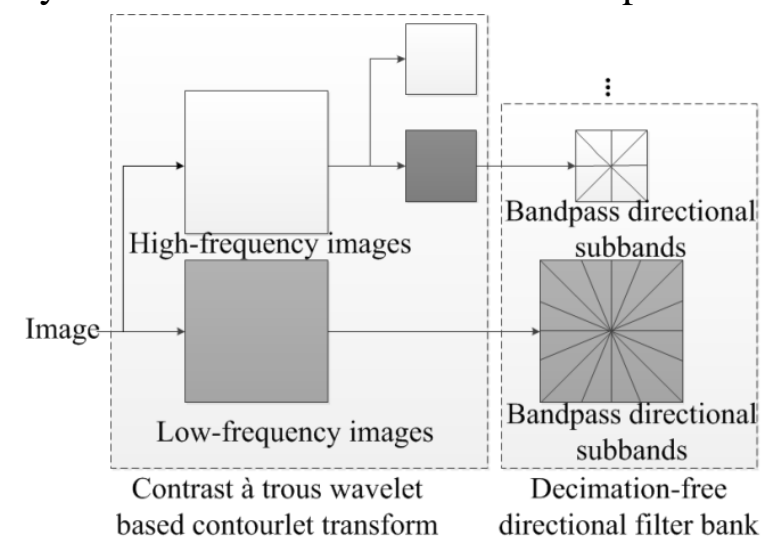

Fig. 4 The flowchart of contrast à trous wavelet based contourlet transform.

Fig. 4 shows the flowchart of the proposed sparse representation algorithm: contrast à trous wavelet based contourlet transform. It can be observed from Fig. 4 that the sub-sampling operations are eliminated. The algorithm consists of two parts. One is the contrast à trous wavelet transform, and the other is a decimation-free directional filter bank. As a whole, 
using the contrast à trous wavelet based contourlet transform will be helpful in capturing substantial geometrical structures of prostate MR images, which will be demonstrated in the experiments later in this paper.

\section{The Marker-based Watershed Segmentation Using Texture Gradient}

The existence of a large number of minute details in the image results in the edge line buried in disorderly watershed line, especially for texture of images. However, texture is regularly located and homogenous at visual effects. The ideal segmentation method should divide the regions with the same texture features into one part. Hence, a texture gradient feature which can highlight the texture boundary and minimize the inner of the texture image is expected.

In this section, the texture gradient is combined with marker-based watershed to reduce the number of segmented regions. First, the modified gradient images are generated according to the definition of texture gradient. Then, marker-based watershed algorithm is used to segment the modified gradient images.

\subsection{Texture Gradient}

A simple method of obtaining texture gradient is to calculate the gradient of the amplitude in each subband and add these gradients. But it is noteworthy that while the texture feature of original image is amplified, the small boundary is enlarged, whose texture may cause narrow region around double-edge $\mathrm{e}^{22}$. In this paper, in order to avoid the narrow region, one-dimensional median filter is performed along the horizontal and vertical directions for the amplitude of each subband, respectively. The filter suppresses over-segmentation caused by double-edge and smoothes texture feature.

\subsubsection{Feature Gradient Computation}

The texture of original image can be represented as $T(x, y)$, and $M T(x, y)$ is defined as follows:

$$
M T_{i}(x, y)=\text { MedianFilter }\left(T_{i}(x, y)\right), 1<i \leq n .
$$

where $n$ is the number of subbands, the length of the one-dimensional median filter is $2^{(l+2)}$. The texture gradient ${ }^{23} T G(x, y)$ can be calculated by:

$$
T G(x, y)=\sum_{i=1}^{n} \frac{\left|\nabla\left(M T_{i}(x, y)\right)\right|}{l_{2}\left(M T_{i}\right)}
$$

where $n$ is the number of subbands, $\nabla$ is approximated using a Gaussian gradient calculation algorithm (with the scale parameter $\sigma$ set to 2.0). $T_{i}(x, y)$ is the amplitude of the subband $i$ decomposed by complex wavelet transform. $l_{2}\left(M T_{i}\right)$ is the $l_{2}$ norm energy of $M T_{i}$, which can normalize the texture gradient of each subband. 


\subsubsection{The Modified Gradient Images}

In this paper, the complex wavelet is replaced by the ratio of low-pass pyramid. The ratio of low-pass pyramid coincides with human vision system (HVS). At the same time, the modified transform algorithm can efficiently characterize the texture gradient.

The final modified gradient can be calculated as follows:

$$
G(x, y)=\frac{\operatorname{mix} \times(\nabla f)^{1.2}}{(F(|M T(x, y)|))^{3}}+(T G(x, y))^{3}
$$

where $\nabla f$ is just the gradient of the original intensity image, mix is a constant coefficient, set to weigh the intensity and texture gradients. $F(|M T(x, y)|)$ is designed as:

$$
F(|M T(x, y)|)= \begin{cases}\operatorname{mean}(M T) & \text { if }|M T(x, y)| \leq \text { mean }|M T| \\ (|M T(x, y)|) & \text { if }|M T(x, y)|>\text { mean }(|M T|)\end{cases}
$$

The meaning of the parameters within the definition $G(x . y)$ is justified as follows:

(1) $(\nabla f)^{1.2}$ : This is used to emphasize the weight of gradient values by expending the range of the gradient image.

(2) $T G(x, y)^{3}$ : This is set to emphasize large texture gradient values to highlight the texture boundaries.

(3) $(F(M T(x, y)))^{3}$ : This is included to reduce the texture values within texture regions.

The modified gradient images can efficiently characterize the texture gradient feature, which can highlight the texture boundary and minimize the inner of the texture image. Then, marker-based watershed algorithm is used to segment the modified gradient images.

\subsection{Marker-based Watershed Algorithm}

One of the effective methods of restraining over-segmentation of watershed is to pre-process the raw image data. Marker-based watershed segmentation takes that strategy and reduces the number of segmented regions.

Marker-based watershed submerges images from some special pixels such as local minimum, and accurately locates homogeneous regions with large size in textures, density and other features of images through mark recognition algorithm ${ }^{24}$. The segmentation method follows three steps as follows: (1) Seek a small initial region as seed point to start submerge images; (2) Calculate threshold values which changes with different image pixel values; (3) Perform region growing segmentation using the seed point and threshold.

In the method proposed in this paper, marker-based watershed algorithm is used to segment the modified gradient images referred in section 3.1. First, some threshold values are set to get a series of binary images, and an acceptable minimum size is set to ensure the size of segmented regions is not too small. Then, these binary images are segmented and the regions with bigger size than minimum size are reserved. Finally, these binary images are marked and performed the watershed segmentation to generate the segmentation results. 


\section{Algorithm and Experiment}

The proposed method is evaluated by a series of experiments. The primary procedures of the integration of marker watershed segmentation and modified contourlet transform include three steps. First, original images are performed the decomposition of contrast à trous wavelet based contourlet transform. Then, the low-frequency images produced in the decomposition are segmented by marker-based watershed segmentation. Finally, the inverse contourlet transform is implemented to get high resolution image. The achieved image is the final segmentation results.

The experimental data is a set of T1-weighted MR prostate images(spin echo, $\mathrm{TR}=2324 \mathrm{~ms}$, $\mathrm{TE}=75 \mathrm{~ms}$, flip angle $=90,528 \times 528$ matrix, $1.5 \mathrm{~T}$ magnetic field strength, $6 \mathrm{~mm}$ thick slices, FFS patient position). Each image is collected on a PHILIPS-FE9A9DC Nuclear Magnetic Resonance Spectrometer with same parameters, in-plane image size of $528 \times 528$ and pixel size of $0.78 \times 0.78 \mathrm{~mm} 2$. The manual segmentation of each image is also provided by a clinical expert. The computer used for all tests has an Intel Core i3-2120, 3.3-GHz 32-b Quad-Core processor with 8192-MB RAM.

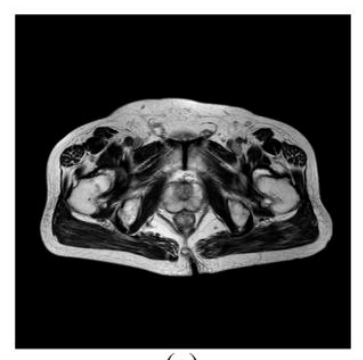

(a)

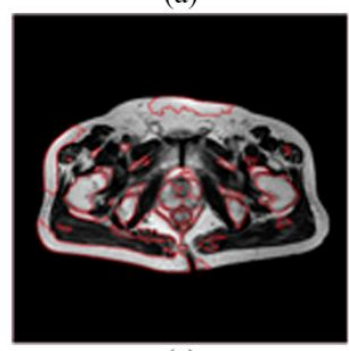

(c)

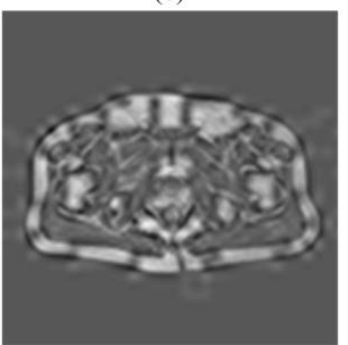

(e)

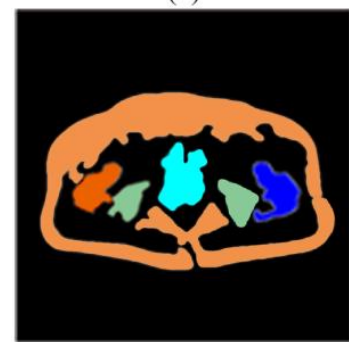

(g)

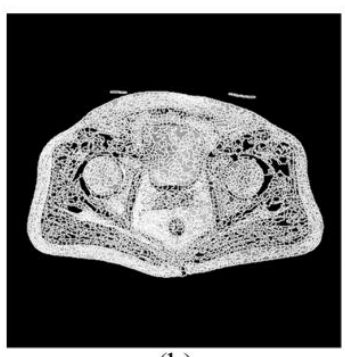

(b)

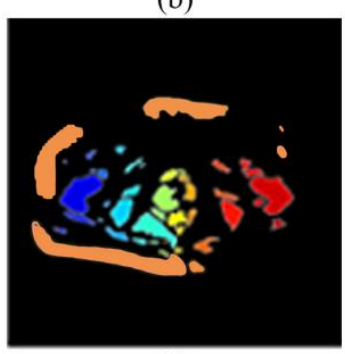

(d)

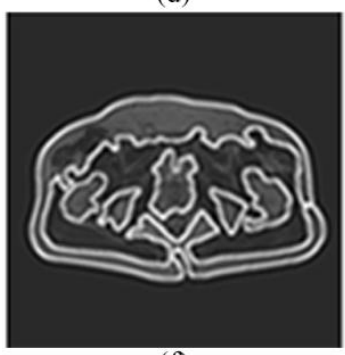

(f)

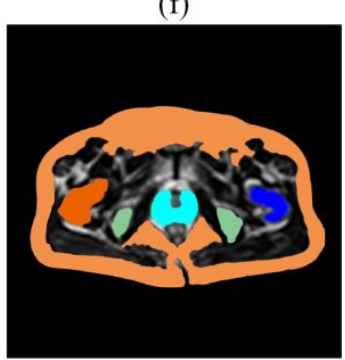

(h) 
Fig. 5 The comparison among the effects of different methods of an prostate MR image: (a) Original MR prostate image, (b) The segmentation result using traditional watershed directly. The segmentation result contains so many tiny regions that regions of interest cannot be identified easily. (c) The segmentation using marker-based watershed only. (c) is labeled with different numbers and coloured by different colors, and (d) is the result of the coloration. In (c) and (d), segmentation result of marker-based watershed algorithm is comprehensively presented. Although the number of segmented regions is reduced, small regions, which are not the main tissues, still exist. Additionally, The segmentation result does not obtain integrately main organs in image (a). (e) Image segmentation result of marker-based watershed using traditional contourlet transform. The result contains main tissues, but the clarity is decreased. (f)-(g) Segmentation results of the proposed method. (f) is the segmentation result of the proposed method in this paper. In order to make the organs intuitive in two-dimentional images and the reconstructon model, (f) is labeled with different numbers and coloured by different colors, and ( $\mathrm{g}$ ) is the result of coloration. (h) Hand-labeled segmentation result.

Fig. 5 shows a detailed experiment of the prostate images. It can be observed from Fig. 5 that the proposed method restrains the number of segmented regions. The segmentation results correctly correspond to the main tissues in the images, and each tissue is integrally segmented respectively with the elimination of small regions. At the same time, the proposed method also can keep a high definition of images. The achieved results are more continuous and satisfying as a result of the human organs.

The experimental results of other prostate images are illustrated in Fig. 6. Desirable experimental results were achieved with the proposed method. 


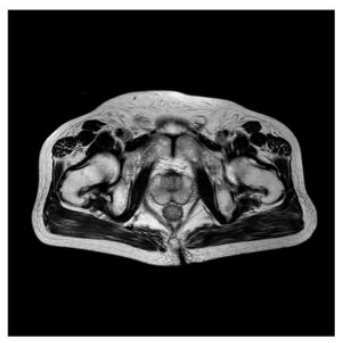

(a)

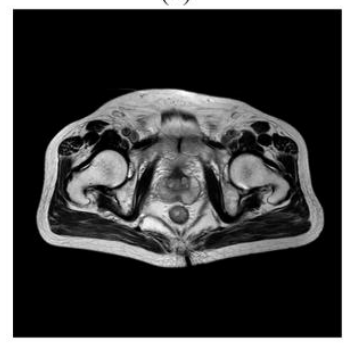

(e)

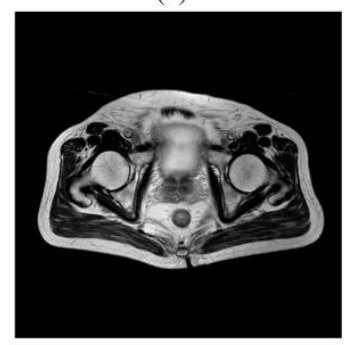

(i)

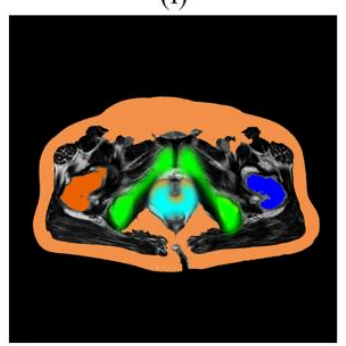

(m)

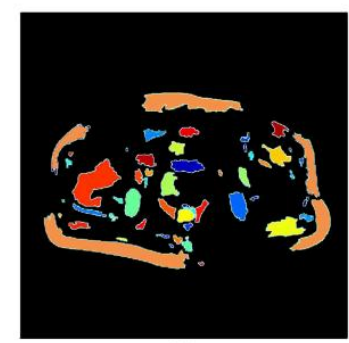

(b)

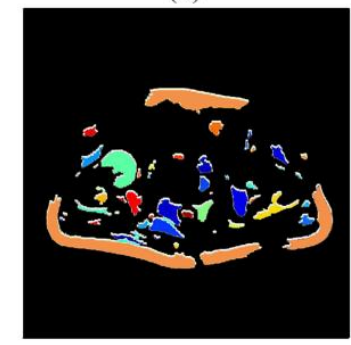

(f)

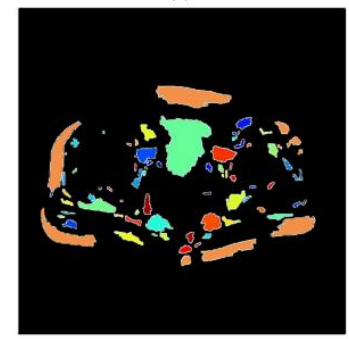

(j)

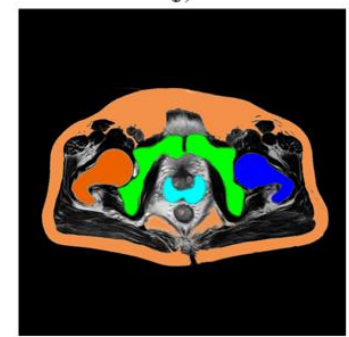

(n)

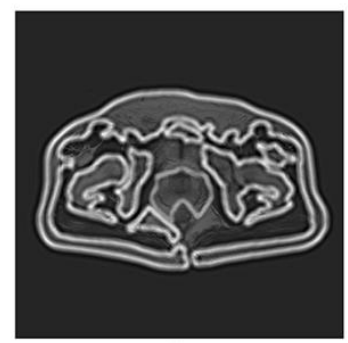

(c)

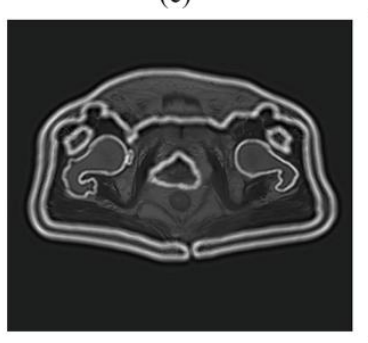

(g)

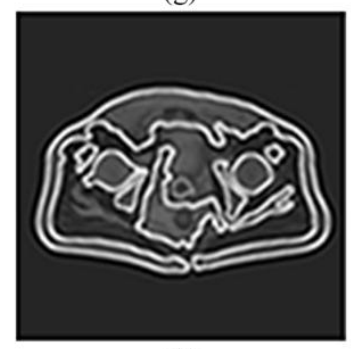

(k)

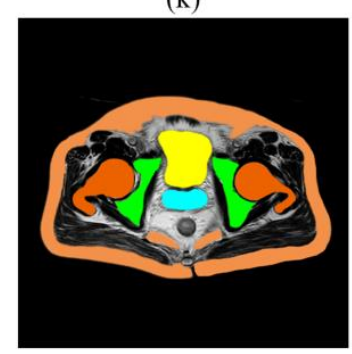

(o)

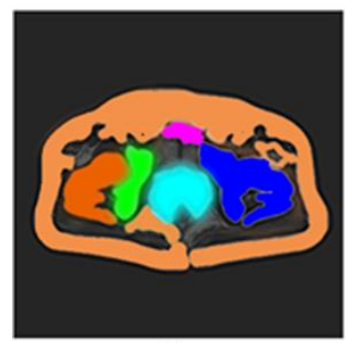

(d)

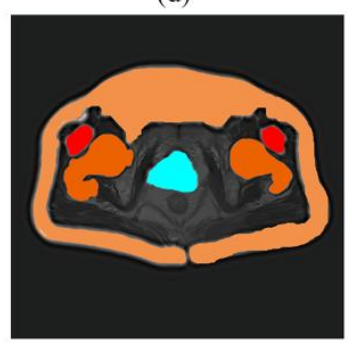

(h)

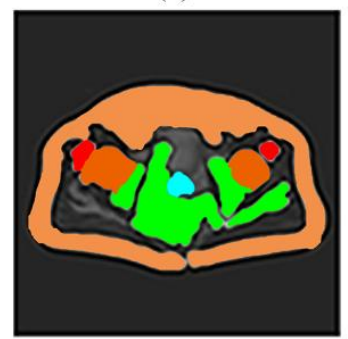

(1)

Fig. 6 Segmentation results of several methods: (a) Original MR prostate image. (b) The watershed segmentation result of marker-based watershed. (c)-(d) Segmentation results of the proposed method of image shown in (a). (e) Original MR prostate image. (f) The watershed segmentation result of (e). (g)-(h) Segmentation results of the proposed method of image displayed in (e). (i) Original MR prostate image. (j) The watershed segmentation result of (i). (k)-(1) Segmentation results of the proposed method shown in original image (i). (m) Hand-labeled segmentation result of (a). (n) Hand-labeled segmentation result of (e). (o) Hand-labeled segmentation result of (i).

Six images, which are in the same slice with Fig. 5 (a) and contain similar contents, are segmented by traditional watershed method, marker-based watershed algorithm, marker-based watershed combining traditional contourle transform and the proposed method. The segmentation results are compared and the average datas of the segmentation of six prostate MR images are listed in Table 1. 
Table 1 The comparison among different algorithms

\begin{tabular}{|c|c|c|c|}
\hline $\begin{array}{l}\text { The way obtaining } \\
\text { segmentation results }\end{array}$ & $\begin{array}{l}\text { The number of } \\
\text { segmented regions }\end{array}$ & $\begin{array}{l}\text { The accuracy rate of } \\
\text { each way }\end{array}$ & $\begin{array}{l}\text { The number of correctely } \\
\text { segmented organs }\end{array}$ \\
\hline $\begin{array}{l}\text { Watershed method } \\
\text { only }\end{array}$ & $>200$ & - & - \\
\hline $\begin{array}{l}\text { Marker-based } \\
\text { watershed algorithm }\end{array}$ & 34 & 0.6214 & 4 \\
\hline $\begin{array}{l}\text { Marker-based } \\
\text { watershed combining } \\
\text { traditional contourle } \\
\text { transform }\end{array}$ & 12 & 0.7635 & 6 \\
\hline The proposed method & 6 & 0.8729 & 6 \\
\hline Handle-labeled result & 6 & - & - \\
\hline
\end{tabular}

The comparisons among different approaches are summarized in Table 1. The accuracy rate of segmentation results is calculated by the comparison pixel by pixel with the hand-labeled segmentation results, which are all labeled and binarized. It can be observed that the proposed method has higher segmentation accuracy rate than watershed and marker-based watershed. In Table 1, there are six organs in the given slice, so ideal number of segmented regions is six. The number is counted following the principle: if a segmented organ has $80 \%$ pixels same with the handle-labeled result, the organ is counted as a correct segmentation organ. It can be observed from Table 1 that the proposed method consistently restrains the number of segmented regions and generates correct number of segmented regions.

Table 2 The average accuracy rate of each main organ generated by the compared algorithms. The average accuracy rate of each main organ generated by marker-based watershed algorithm, marker-based watershed combining traditional contourle transform and the proposed method is represented as mean \pm standard deviation $[1 \%]$.

\begin{tabular}{lllllll}
\hline $\begin{array}{l}\text { Main organs in } \\
\text { prostate MR } \\
\text { images }\end{array}$ & $\begin{array}{l}\text { The average } \\
\text { percentage of the } \\
\text { volume in the } \\
\text { target organs }\end{array}$ & $\begin{array}{l}\text { Watershe } \\
\text { d methody }\end{array}$ & $\begin{array}{l}\text { Marker-based } \\
\text { watershed } \\
\text { algorithm }\end{array}$ & $\begin{array}{l}\text { Marker-based } \\
\text { watershed combining } \\
\text { traditional contourle } \\
\text { transform }\end{array}$ & $\begin{array}{l}\text { The } \\
\text { method }\end{array}$ \\
\hline Skin & 0.5976 & - & $62.16 \pm 6.83$ & $76.23 \pm 5.42$ & $90.15 \pm 3.53$ \\
\hline Left femur bone & 0.0866 & - & $62.17 \pm 7.21$ & $79.36 \pm 4.67$ & $82.17 \pm 5.49$ \\
\hline Right femur bone & 0.0814 & - & $66.29 \pm 4.31$ & $72.39 \pm 5.39$ & $86.83 \pm 5.91$ \\
\hline Pelvis & 0.1513 & - & $59.17 \pm 7.69$ & $80.19 \pm 7.56$ & $81.99 \pm 9.17$ \\
\hline Prostate & 0.0358 & - & $66.12 \pm 6.37$ & $69.16 \pm 8.72$ & $82.57 \pm 7.76$ \\
\hline Urinary bladder & 0.0473 & - & $61.17 \pm 9.34$ & $72.32 \pm 4.28$ & $81.86 \pm 5.62$ \\
\hline
\end{tabular}

The average accuracy rate of each main organ generated by the compared algorithms are provided in Table 2, and the number in brackets is the responding standard deviation. The average percentage of the volume in the target organs is also represented. The accuracy rate of each algorithm in Table 1 is the weighted average of the accuracy rate of main targets, and 
each accuracy rate is weighted by the average percentage of the volume in the target organs. Table 2 shows that the proposed method has higher segmentation accuracy rate than watershed and marker-based watershed.

The experimental results suggest that the improved watershed algorithm can generate more satisfactory results by the combination of à trous wavelet based contourlet transform. The main organs in the segmented image are segmented integrated and the over-segmentation is restrained.

\section{Conclusion}

This work proposes the contrast à trous wavelet based contourlet transform and uses it to produce an effective watershed segmentation technique for prostate MR images. The contrast à trous wavelet based contourlet transform can obtain sparse representation and capture substantial geometrical structures of prostate MR images. Additionally, the "texture gradient" is defined, and the texture gradient is combined with marker-based watershed algorithm to reduce the number of segmented regions. According to the proposed method, original images are performed the decomposition of contrast à trous wavelet based contourlet transform. Then, the low-frequency images produced in the decomposition are segmented by marker-based watershed segmentation. Finally, the inverse contourlet transform is implemented to get high resolution image. The achieved image is the final segmentation results.

The proposed method is tested by 36 prostate MR images and compared with several image segmentation algorithms; the experiment and comparison results show that the proposed method consistently restrains the number of segmented regions. The segmentation results correctly correspond to the main tissues in the images, and each tissue is integrally classified respectively with the elimination of small regions. Additionally, the segmentation accuracy rate is $87.29 \%$, which is higher than other methods under comparison.

The main drawback of the proposed algorithm is that the segmentation results are not accurate enough to be used for clinical practice. This problem may be alleviated by other published methods for over-segmentation removal. Using those methods to improve watershed following the proposed framework, and making comparison among the improved methods will be useful to enhance the accuracy rate of each target.

\section{Acknowledgments}

We gratefully acknowledge our research team at the Center for Advanced Mechanisms and Robotics, Tianjin University and the help of the Tianjin Medical University Cancer Institute and Hospital. This research is partly supported by National Science Foundation of China (Grant No. 51175373), Education Program for New Century Excellent Talents (NCET-10-0625), Key Technology and Development Program of the Tianjin Municipal Science and Technology Commission (12ZCDZSY10600).

\section{Reference}


1. X. Wu, F. Zhang, R. Chen, W. L. Zheng and X. M. Yang, "Recent advances in imaging-guided interventions for prostate cancers," Cancer Lett. 349(2), 114-119 (2014) [doi:10.1016/j.canlet.2014.04.015].

2. S. Saito, K. Ito, A. Yorozu, M. Aoki, H. Koga, T. Satoh, T. Ohashi, N. Shigematsu, S. Maruo, T. Kikuchi, S. Kojima, T. Dokiya, M. Fukushima and H. Yamanaka, "Nationwide Japanese Prostate Cancer Outcome Study of Permanent Iodine-125 Seed Implantation (J-POPS)," Int. J. Clin. Oncol. 20(2), 375-385 (2015) [doi:10.1007/s10147-014-0704-4].

3. C. Ménard, R. C. Susil, P. Choyke, G. S. Gustafson, W. Kammerer, H. Ning, R. W. Miller, K. L. Ullman, N. S. Crouse and S. Smith, "MRI-guided HDR prostate brachytherapy in standard 1.5 T scanner," Int. J. Radiat. Oncol. 59(5), 1414-1423 (2004) [doi:10.1016/j.ijrobp.2004.01.016].

4. Y. Guo, Y. Gao, Y. Shao, T. Price, A. Oto and D. Shen, "Deformable segmentation of 3D MR prostate images via distributed discriminative dictionary and ensemble learning," Med. Phys. 41(7), (2014) [doi:10.1118/1.4884224].

5. H. Masoumi, A. Behrad, M. A. Pourmina and A. Roosta, "Automatic liver segmentation in MRI images using an iterative watershed algorithm and artificial neural network," Biomed. Signal Proces. 7(5), 429-437 (2012) [doi:10.1016/j.bspc.2012.01.002].

6. E. A. Zanaty and A. Afifi, "A watershed approach for improving medical image segmentation," Computer Methods In Biomechanics And Biomedical Engineering 16(12), 1262-1272 (2013) [doi:10.1080/10255842.2012.666794].

7. F. Malmberg and C. L. L. Hendriks, "An efficient algorithm for exact evaluation of stochastic watersheds," Pattern Recogn. Lett. 47(80-84 (2014) [doi:10.1016/j.patrec.2014.03.016].

8. L. Patino, "Fuzzy relations applied to minimize over segmentation in watershed algorithms," Pattern Recogn. Lett. 26(6), 819-828 (2005) [doi:10.1016/j.patrec.2004.09.036].

9. W. Wei and Y. Xin, "Rapid, man-made object morphological segmentation for aerial images using a multi-scaled, geometric image analysis," Image Vision Comput. 28(4), 626-633 (2010) [doi:10.1016/j.imavis.2009.10.002].

10. R. Gaetano, G. Masi, G. Poggi, L. Verdoliva and G. Scarpa, "Marker-Controlled Watershed-Based Segmentation of Multiresolution Remote Sensing Images," IEEE Trans. Geosci. Remote Sensing 53(6), 2987-3004 (2015) [doi:10.1109/tgrs.2014.2367129].

11. M. N. Do and M. Vetterli, "The contourlet transform: An efficient directional multiresolution image representation," IEEE T. Image Process. 14(12), 2091-2106 (2005) [doi:10.1109/tip.2005.859376].

12. M. N. O. Sadiku, C. Akujuobi and R. C. Garcia, "An introduction to wavelets in electromagnetics," IEEE Microw. Mag. 6(2), 63-72 (2005) [doi:10.1109/mmw.2005.1491268].

13. M. N. Do and M. Vetterli, "Contourlets: a new directional multiresolution image representation," in Signals, Systems and Computers, 2002. Conference Record of the Thirty-Sixth Asilomar Conference on, pp. 497-501, IEEE (2002)

14. A. Toet, "Hierarchical image fusion," Mach. Vision Appl. 3(1), 1-11 (1990)

15. L. G. Y. Wanhai, "A Multiscale Contrast-Pyramid-Based Image Fusion Scheme and Its Performance Evaluation [J]," Acta Optica Sinica 11(012 (2001) 
16. L. Yang, B. Guo and W. Ni, "Multimodality medical image fusion based on multiscale geometric analysis of contourlet transform," Neurocomputing 72(1), 203-211 (2008) [doi:10.1016/j.neucom.2008.02.025].

17. S. Paris, S. W. Hasinoff and J. Kautz, "Local Laplacian Filters: Edge-aware Image Processing with a Laplacian Pyramid," ACM Trans. Graph. 30(4), 11 (2011) [doi:10.1145/1964921.1964963].

18. D. Y. Hyun, J. H. Heu, C. S. Kim and S. U. Lee, "Prioritized image and video colorization based on Gaussian pyramid of gradient images," J. Electron. Imaging 21(2), 14 (2012) [doi:10.1117/1.jei.21.2.023027].

19. M. González - Audícana, X. Otazu, O. Fors and A. Seco, "Comparison between Mallat's and the 'à trous' discrete wavelet transform based algorithms for the fusion of multispectral and panchromatic images," Int. J. Remote Sens. 26(3), 595-614 (2005) [doi:10.1080/01431160512331314056].

20. F. Wegner, M. Both and R. Fink, "Automated detection of elementary calcium release events using the à trous wavelet transform," Biophys. J. 90(6), 2151-2163 (2006) [doi:10.1529/biophysj.105.069930].

21. S. H. Chen, H. B. Su, J. Tian and C. S. Zhan, "Best tradeoff for remote sensing image fusion based on three-dimensional variation an a trous wavelet," J. Appl. Remote Sens. 7(18 (2013) [doi:10.1117/1.jrs.7.073491].

22. R. J. O'Callaghan and D. R. Bull, "Combined morphological-spectral unsupervised image segmentation," IEEE T. Image Process. 14(1), 49-62 (2005) [doi:10.1109/tip.2004.838695].

23. P. R. Hill, C. N. Canagarajah and D. R. Bull, "Image segmentation using a texture gradient based watershed transform," IEEE T. Image Process. 12(12), 1618-1633 (2003) [doi:10.1109/tip.2003.819311].

24. W. Zhang and D. Jiang, "The marker-based watershed segmentation algorithm of ore image," in Communication Software and Networks (ICCSN), 2011 IEEE 3rd International Conference on, pp. 472-474, IEEE (2011) [doi:10.1109/ICCSN.2011.6014611].

\section{$\underline{\text { Caption List }}$}

Fig. 1 Over-segmentation caused by watershed.

Fig. 2 Flowchart of the proposed segmentation method.

Fig. 3 Flowchart of contourlet transform.

Fig. 4 The flowchart of contrast à trous wavelet based contourlet transform.

Fig. 5 The comparison among the effects of different methods of an prostate MR image.

Fig. 6 Segmentation results of several methods. 
Table 1 The comparison among different algorithms.

Table 2 The average accuracy rate of each main organ generated by the compared algorithms. 